\title{
Improving thermal substation inspections utilising machine learning
}

Link to publication record in Manchester Research Explorer

\section{Citation for published version (APA):}

Straker, A., Carrasco, J., Podd, F., Gardner, R., \& Cotton, I. (2019). Improving thermal substation inspections utilising machine learning. 1-9. Paper presented at Thermosense: Thermal Infrared Applications XLI, Baltimore, United States.

\section{Citing this paper}

Please note that where the full-text provided on Manchester Research Explorer is the Author Accepted Manuscript or Proof version this may differ from the final Published version. If citing, it is advised that you check and use the publisher's definitive version.

\section{General rights}

Copyright and moral rights for the publications made accessible in the Research Explorer are retained by the authors and/or other copyright owners and it is a condition of accessing publications that users recognise and abide by the legal requirements associated with these rights.

\section{Takedown policy}

If you believe that this document breaches copyright please refer to the University of Manchester's Takedown Procedures [http://man.ac.uk/04Y6Bo] or contact uml.scholarlycommunications@manchester.ac.uk providing relevant details, so we can investigate your claim.

\section{OPEN ACCESS}




\section{Improving thermal substation inspections utilising machine learning}

\section{Alastair Straker, Joaquin Carrasco, Frank Podd, Richard Gardner, Ian Cotton}

Alastair Straker, Joaquin Carrasco, Frank Podd, Richard Gardner, lan Cotton, "Improving thermal substation inspections utilising machine learning," Proc. SPIE 11004, Thermosense: Thermal Infrared Applications XLI, 1100406 (2 May 2019); doi: 10.1117/12.2518953

Event: SPIE Defense + Commercial Sensing, 2019, Baltimore, Maryland, United States 


\title{
Improving thermal substation inspections utilising machine learning
}

\author{
Alastair Straker ${ }^{\mathrm{a}}$, Joaquin Carrasco ${ }^{\mathrm{b}}$, Frank Podd ${ }^{\mathrm{c}}$, Richard Gardner ${ }^{\mathrm{d}}$, and Ian Cotton ${ }^{\mathrm{e}}$ \\ a,b,c,d,e University of Manchester, Sackville Street, Manchester, UK
}

\begin{abstract}
Periodic thermal imaging inspection of air-insulated substations can lead to false negatives due to the heating effects of solar radiation and cooling effects of wind and precipitation. This work aims to characterise the effects of wind on thermal images of thermally loaded equipment, allowing thermal response forecasts to be made.

Data is collected in two load patterns from an indoors experiment, comprising a current loop of two overheadline conductors energised by a high-current DC power supply. Wind is emulated by an industrial fan. Infrared images, environmental data (ambient temperature, humidity, pressure, wind speed and direction) and electrical load data are all captured periodically. A further dataset from an in-service substation is used.

Models are created using vector autoregressive and long short-term memory recurrent neural network models in order to further develop the methods presented by Bortoni et al. The results display a clear improvement over those found in the literature, highlighting the utility of modern data-processing techniques. These results present an opportunity to extract meaningful information for long term thermal condition monitoring of power substations.
\end{abstract}

Keywords: thermography, substation, inspection, LSTM, machine learning, regression

\section{INTRODUCTION}

Air-insulated power substations are facilities for the distribution and management of electrical power. They vary in size and function but are invariably hazardous environments. High load on substation componentry means that accelerated degradation can be catastrophic. Other risks include lethal electric shock. In order to mitigate the risks regular inspections are carried out, based on the methodology of condition-based monitoring. Thermal imaging inspection is one of the inspection modalities used. Thermal images convey only the surface temperature of equipment to inspection engineers. This is affected by various conditions, including the equipment load, environmental conditions and emmissivity. Hot-spots, a common precursor to faults, are most visible under heavy electrical load. A piece of equipment under heavy load, in cold and windy conditions may not appear to be heating significantly when thermally inspected, for example, therefore increasing the chance that a false-negative occurs. This work assumes emmissivity is constant throughout testing though acknowledges there is scope for including it in future work. This work seeks to use regression based data analysis techniques to model the effects of these external factors, thus improving inspection accuracy.

\section{THERMAL SUBSTATION INSPECTION}

There are over 300 substations in the UK and inspecting them requires specialist knowledge of their operation. Engineers walk around the facilities with sensors, taking measurements as they go. They then use these measurements and the context within which they are taken to make subjective judgements on the health of the asset. Often engineers rely on comparisons between the three phases to determine if a fault might be present. The specialist knowledge and access to the equipment means that there are only a small number of engineers qualified to make these judgements and due to this and the large number of facilities, inspections are generally completed on a three-monthly basis. This limitation on when inspections may be completed means that there is no way to control the environmental or load conditions the measurements are taken in.

Further author information: (Send correspondence to A. Straker)

A. Straker: E-mail: alastair.straker@postgrad.mancheser.ac.uk

Thermosense: Thermal Infrared Applications XLI, edited by Jaap de Vries,

Beate Oswald-Tranta, Proc. of SPIE Vol. 11004, 1100406 · C 2019 SPIE

CCC code: $0277-786 \mathrm{X} / 19 / \$ 18 \cdot$ doi: $10.1117 / 12.2518953$

Proc. of SPIE Vol. 11004 1100406-1 


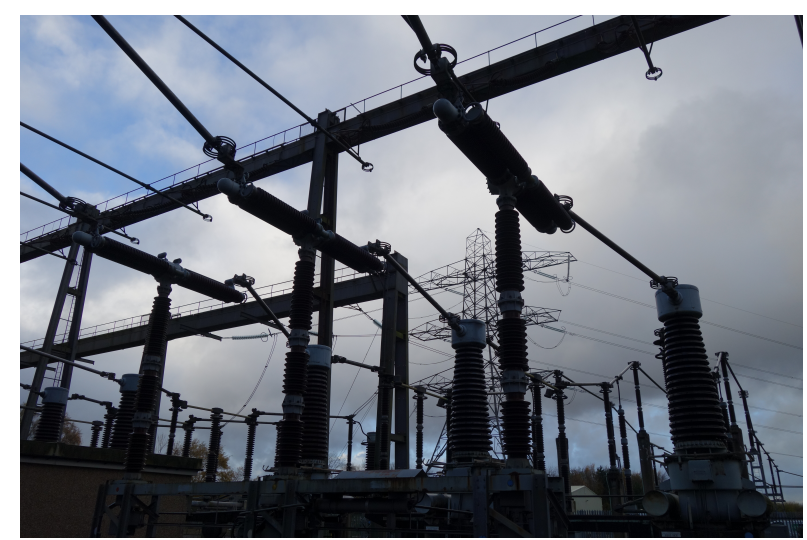

Figure 1. A National Grid Substation in North-West England

There are 3 main sensing modalities used in evaluating asset health during inspection: thermal, partial discharge and gas in oil. Of these, the former two are passive measurements and are both dependent on the environmental conditions. Thermal inspections are the only consideration for this work though there is potential for future expansion into other modalities, using similar data analysis techniques.

The methodology of inspections is condition based monitoring, whereby maintenance is performed on equipment only when fault precursors are detected. This, and the aging power deliver infrastructure in the UK, increases the importance of accurate detection of fault precursors, as only accurate detection ensures critical maintenance is completed.

\subsection{PRIOR WORK}

Most of the literature related to machine learning in substation inspection pertains to classification of faults. Bortoni et $\mathrm{al}^{12}$ and Santos et $\mathrm{al}^{3}$ have completed work towards statistically removing the environmental and load influence on thermal substation inspections. They present schemes for removing the influence analytically and using autoregressive methods. This work seeks to extend their work to modern recurrent neural-network based time series modelling methods, namely long short-term memory (LSTM) neural networks.

\section{DATA ANALYSIS}

\subsection{PREPROCESSING}

The thermal image output from the experiments is preprocessed for readiness for analysis. The preprocessing pipeline is shown in Figure 2. The pipeline reduces the dimensionality of the thermal data and normalises the various inputs.

The average pixel value within the region of interest is then appended to the experiment dataset containing the environmental and load data. A further normalisation step scales values $v$ to: $0 \leq v \leq 1$ then the data is reformatted into vectors of feature values for a given timestep.

\subsection{Vector Autoregressive Model}

A vector autoregressive model equates the output of a system to some combination of coefficients and vectors of inputs. Each input $(X)$ consists of the value $(x)$ at time $t$ multiplied by a coefficient $(\phi)$ and the values at all time steps to time $t-r$, referred to as lagged values. Lagged values of the output are also provided as inputs, defining the model as autoregressive. Therefore, a multivariate vector autoregressive (VAR) model of order 3 $(r=3)$, with three causal variables:

$$
\begin{array}{r}
X_{1, t}=\phi_{11} x_{1, t}+\phi_{12} x_{1, t-1}+\phi_{13} x_{1, t-2}+\phi_{13} x_{t-3,3}, \\
X_{2, t}=\phi_{21} x_{2, t}+\phi_{22} x_{2, t-1}+\phi_{23} x_{2, t-2}+\phi_{23} x_{3, t-3}, \\
X_{3, t}=\phi_{31} x_{3, t}+\phi_{32} x_{3, t-1}+\phi_{33} x_{3, t-2}+\phi_{33} x_{3, t-3}, \\
X_{y, t}=\phi_{y 1} y_{t-1}+\phi_{y 2} y_{t-2}+\phi_{y 3} y_{t-3} .
\end{array}
$$




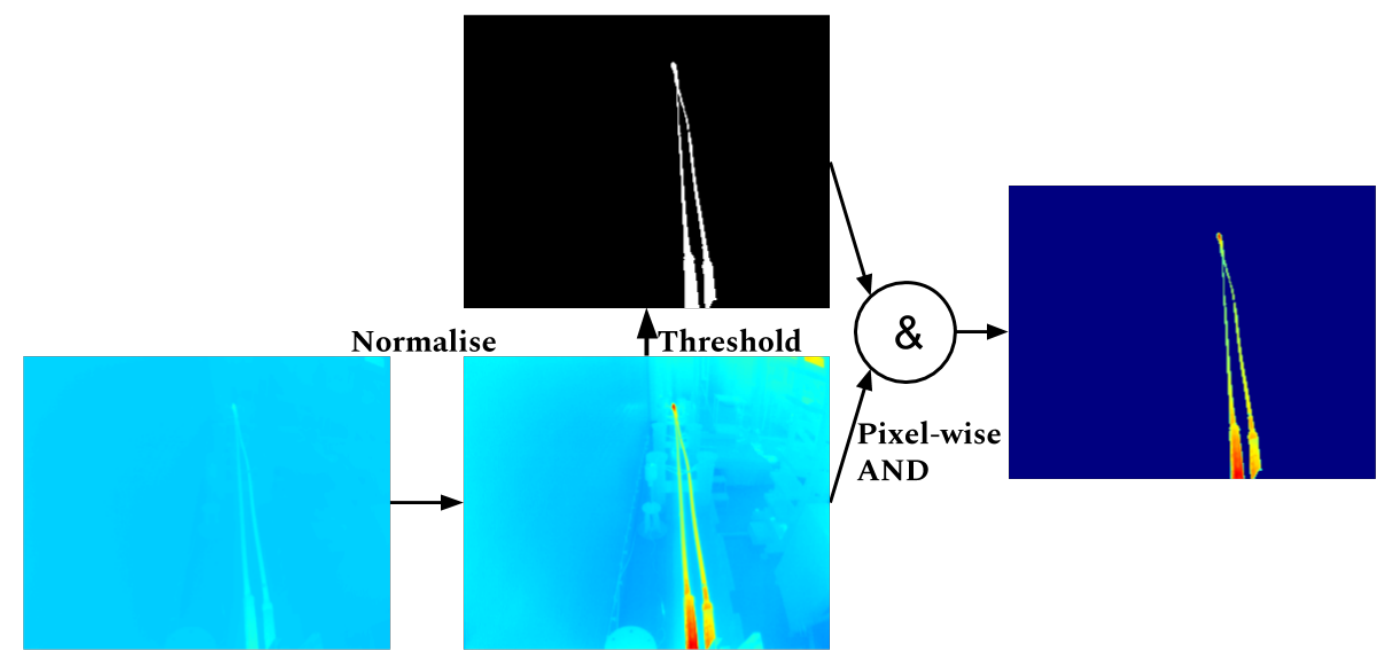

Figure 2. The pre-processing workflow

The output of the system is therefore

$$
Y_{t}=X_{1, t}+X_{2, t}+X_{3, t}+X_{y, t} .
$$

It is optimised using gradient descent. The multivariate lagged values forming the inputs allow the regression to model time-dependant systems more complex than simple linear regression. Bortoni et al use an autoregressive model in 'Infrared thermography applied for outdoor power substations'. ${ }^{3}$

\subsection{Long Short-Term Memory Model}

Long short-term memory (LSTM) neural networks ${ }^{4}$ are a form of recurrent neural network (RNN) pioneered in 1997 by Hochreiter. LSTM networks utilise a data pipeline in their recurrent layer, which allows data to be maintained for long time periods in dataset. This enhances their ability to 'learn' long term causal relationships between data points, without falling victim to exploding or vanishing gradients in the training process. A result of these characteristics is that LSTM networks are suitable for the problem of time-series prediction. Furthermore, being artificial neural networks, they are suitable for scaling up to large numbers of features.

There is no one-size fits all approach to the design of a neural network. The network designer must consider the dataset and what it represents when choosing the architecture. The input dimension of the network is dictated by the number of inputs to the system multiplied by the number of lagged input values to be considered. The output dimension is dictated by the outcome variable, in this case a single continuous value describing the average pixel value of a thermal image.

Two measures were taken in order to reduce the chances of the trained model over-fitting on the training data. First, an L2 regulariser was used. This regulariser ensures that weights within the model decay towards zero, preventing any false emphasis on a single variable. Second, a dropout layer ${ }^{5}$ was added. This layer forces a proportion of the weights in the model to zero during the training process, again preventing over-fitting at the cost of training time.

\section{EXPERIMENTAL DESIGN}

In order to validate the models, an experiment was devised. A pair of overhead-line conductors were electrically loaded, while thermal images were taken of them. An industrial fan was used to emulate the wind and its effects. The electrical current, environmental conditions and thermal images were all recorded to provide datasets with which to create models. A data logging system comprising a FLIR Boson thermal camera, a Vaisala WXT 520 weather station and a Raspberry Pi computer captured thermal images, load levels, environmental conditions (including wind speed) and controls the output load pattern and fan. The setup can be seen in Figure 3. 

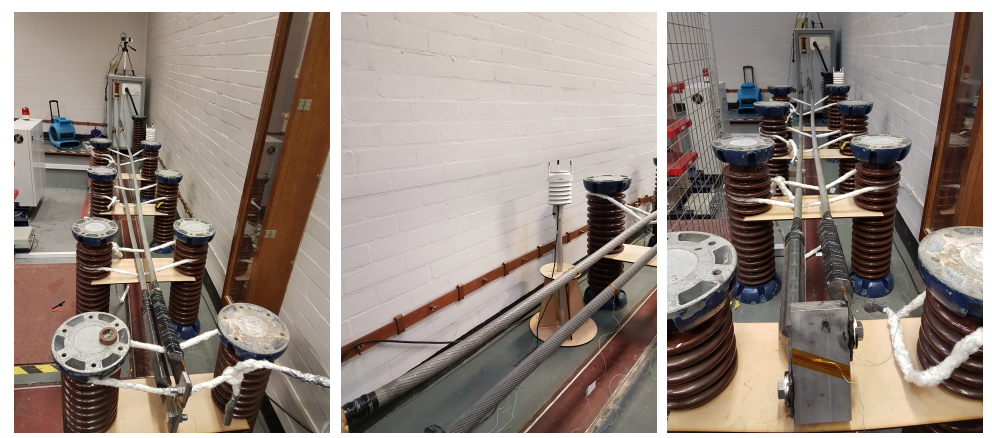

Figure 3. Experimental setup, including power supply, conductors, fan and weather station
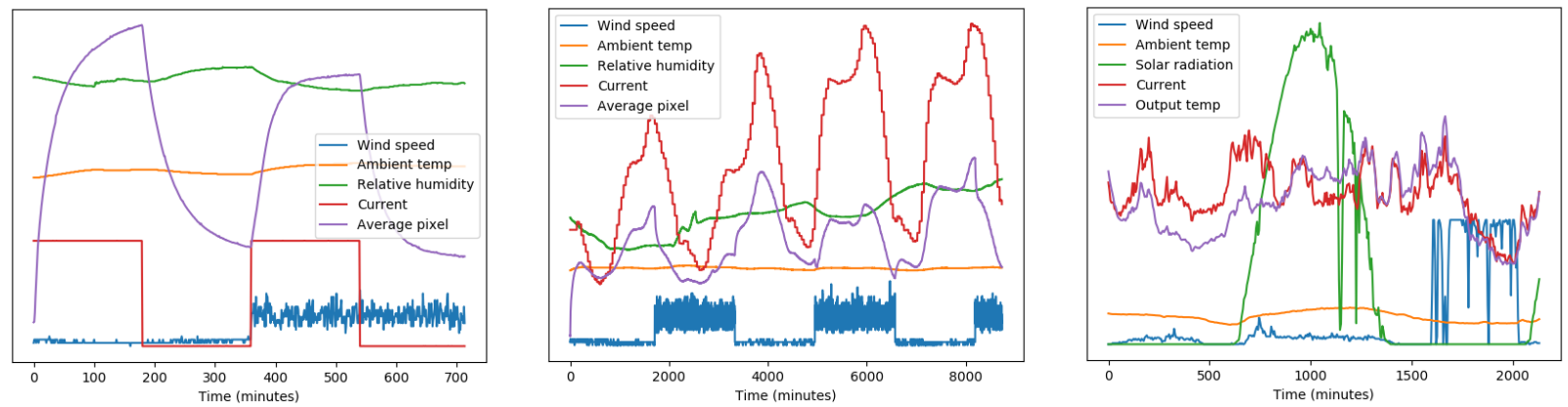

Figure 4. Data from (l-r): step test experiment, realistic experiment, substation field experiment

The resistance of the conductor loop was found experimentally to be $r \approx 0.01 \Omega$. A current range of $0 A \leq I \leq 800 A$ was selected to provide heating of $P \leq 6400 \mathrm{~W}$. Further testing showed this current range to provide sufficient heating for the experiments while remaining under the safe operation temperature threshold of $90{ }^{\circ} \mathrm{C}$.

The experiments were designed to provide data for processing using the two aforementioned algorithms. As such the following requirements were set:

- Provide data such that the time constant of the system $\tau$ can be determined

- Provide data that can approximate real-world conditions

Based on these requirements a pair of experiments were carried out.

To determine the time constant of the system, a current step test was used. The load and wind profiles for this test can be seen in Figure 4. To approximate real-world conditions, a National Grid national loading data was normalised to within safe experimental levels and used to define the load pattern. The load pattern time period was informed by the time constant found in the step test: $\tau \approx 20 \mathrm{~min}$. The industrial fan was enabled in a binary pattern of a differing frequency to the loading data. Models were created based upon these datasets in order to predict the average pixel value of the region of interest based on the load and environmental conditions. A further dataset of equipment temperature (from a thermal image) and environmental conditions from an in-use substation is used to validate that the methodology generalises well to data captured outside of laboratory conditions. This dataset is provided by Bortoni et al and is hereafter referred to as the substation dataset.

\section{RESULTS}

The raw, unprocessed results of the two tests can be seen in Figure 4, alongside the substation dataset. The data have been multiplied by some factor for readability. The presented results represent the best of various attempted algorithm configurations. In the case of the vector-autoregression tests, the only change made to the 
configuration was the number of 'lags' to use. In the case of the LSTM, the number of lags was adjusted, but care also was taken to select the correct number of training epochs, key in preventing over-fitting. Train/test splits of 80:20 were used throughout the analysis. The train dataset is used in the optimisation process, then the model skill is tested on the test dataset. Splitting the dataset as such ensures the model is general and not overfit to the dataset.

Table 1. A summary of $\mathrm{R}^{2}$ Score of the results

\begin{tabular}{|l|l|l|l|}
\hline Dataset & Algorithm & Lags & $\mathrm{R}^{2}$ Score \\
\hline Steptest & VAR & 100 & 0.829 \\
\hline Steptest & LSTM & 1 & 0.999 \\
\hline Realistic & VAR & 10 & 0.953 \\
\hline Realistic & LSTM & 40 & 0.998 \\
\hline Substation & VAR & 10 & 0.891 \\
\hline Substation & LSTM & 10 & 0.977 \\
\hline
\end{tabular}

\section{ANALYSIS}

The results of the models created on the datasets can be seen in Table 1 and Figure 5. The number of lags used in presenting the model is shown, alongside the $\mathrm{R}^{2}$ score for each model. An $\mathrm{R}^{2}$ score can be between zero and one, with the best result being one. Each of the output graphs shows the true output of the training portion of the dataset in blue, with the true output of the test portion of the dataset in orange. The output hypothesis, as generated by each individual model, is shown in green.

Good results are obtained for cases 3-6, corresponding to the larger datasets. It is clear that the modelling skill of both algorithms should theoretically improve with increased datapoints. Both cases 1 and 2 however, demonstrate that the trend of the data is being followed, initially closely, while case 2 maintains a very strong prediction, reflected in its $\mathrm{R}^{2}$ score.

In cases 3 and 4, both algorithms perform well. On average, the LSTM performs more strongly, however it suffers from high frequency oscillations. These are suspected to be symptomatic of the high frequency input wind data, solvable with further pre-processing (in this work only the thermal images are pre-processed) or an adjusted sampling routine. The vector autoregression in case 3 performs well but deviates from the test output. It must be noted that for a general proof of the utility of these algorithms, cases 5 and 6 must be referred to, as they provide more varied environmental conditions, outside of laboratories. Generally, the LSTM network (cases 2, 4 and 6) performs more strongly than the vector autoregression (cases 1, 3 and 5), by visual inspection and by the $\mathrm{R}^{2}$ score. This is especially apparent in the dataset of Prof Bortoni, where the system output is followed closely, including large changes in magnitude and high frequency peaks. Considering the 'in-service' nature of dataset, this result is a particular success. However, the dataset remains limited, with only 430 data points.

In order to further determine the utility of the LSTM method, the results were compared with those presented by Santos et al. ${ }^{3}$ In the absence of a performance metric, a visual analysis of the skill of the methods was performed. The method presented here displays improved performance in both matching the magnitude and location of peaks during prediction. The autoregressive model provided by Bortoni follows the trend well, but data peaks are lagged by $+/$ - a timestep and often incorrect magnitude, demonstrating the improvement in performance from both adapting multivariate and modern LSTM techniques.

A general limitation of the work is the availability of data. Many environmental conditions exist which were not experienced during testing, notably precipitation, and to achieve a truly general model a significant increase in the quantity and variation of the data should be sought out. Furthermore, it is acknowledged that better quality results could be achieved with a calibrated and radiometric thermal camera, allowing temperature measurements to be predicted, rather than pixel intensities. 
(1)

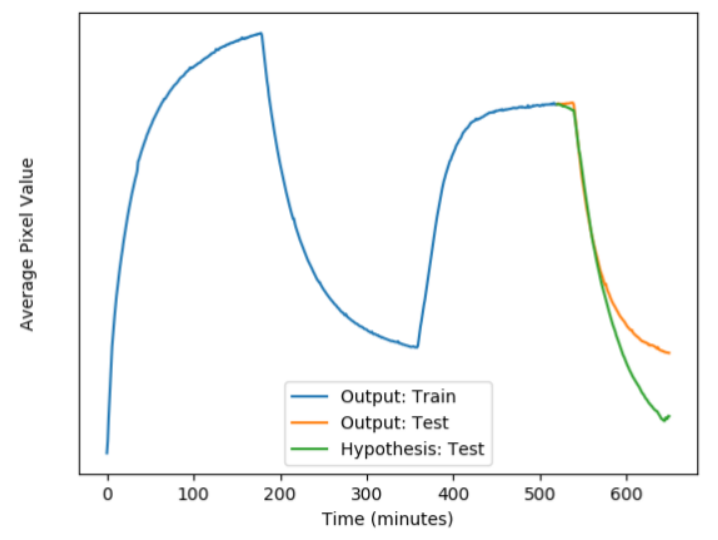

(3)

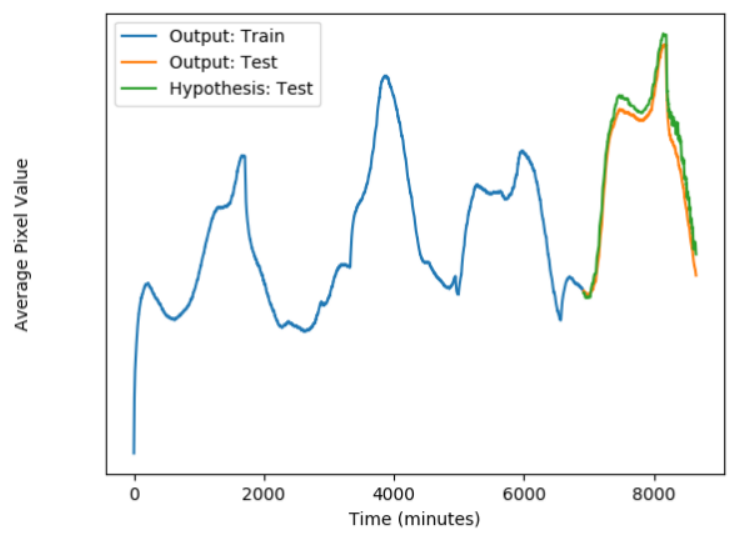

(5)

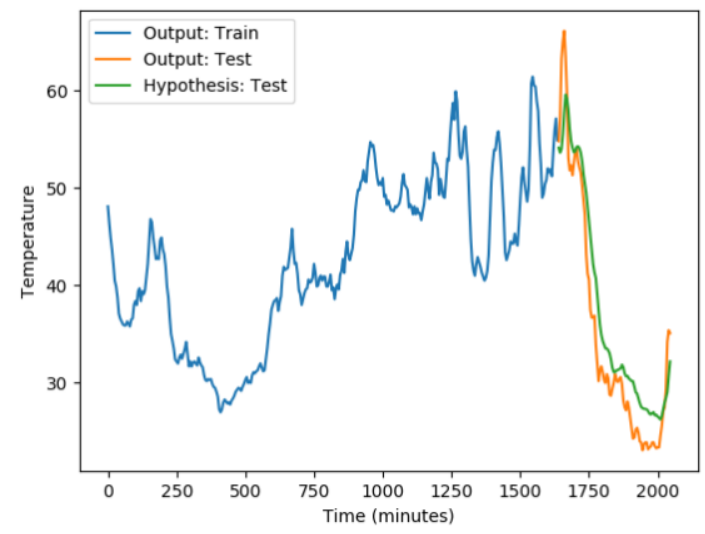

(2)

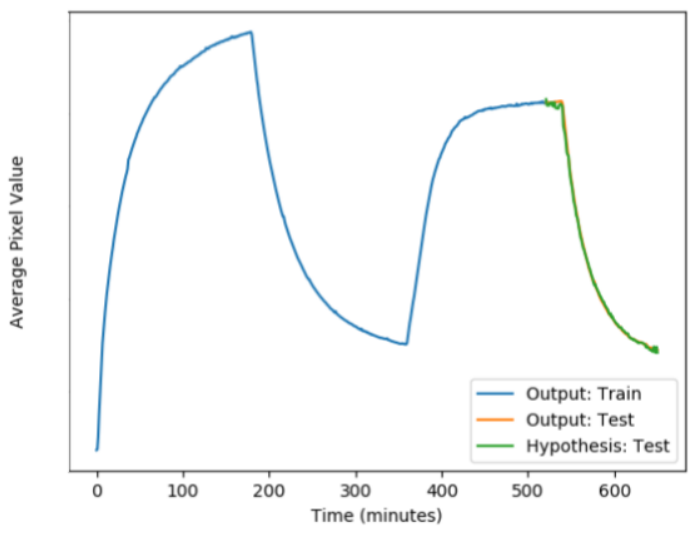

(4)

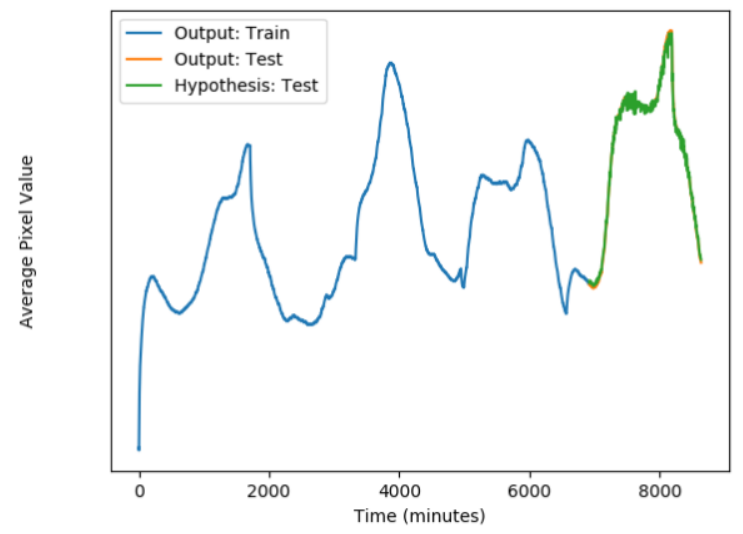

(6)

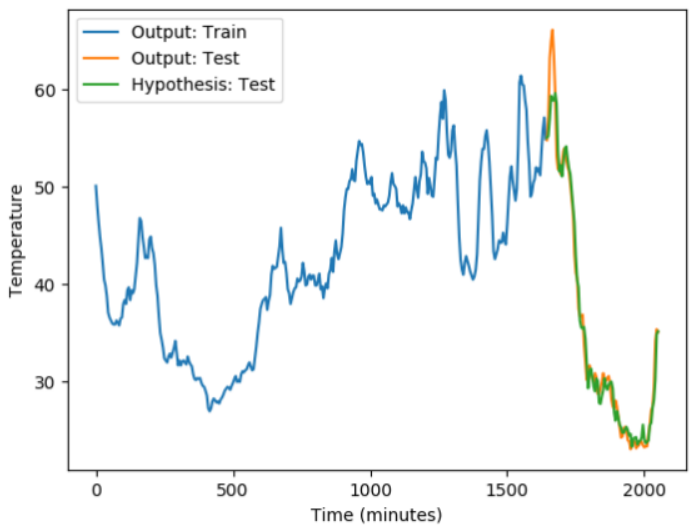

Figure 5. 1, Steptest Autoregression; 2, Steptest LSTM; 3, Realistic Autoregression; 4, Realistic LSTM; 5, Substation Autoregression; 6, Substation LSTM; The y-axis of cases 1-4 is omitted as the system output is a unitless average pixel intensity: proportional to the thermal output in the region of interest 


\section{CONCLUSION}

This paper explores the suitability of a pair of methods for predicting thermal output as detected by a thermal camera, in the context of improving power substation inspections. Data was collected in a series of experiments, forming datasets for characterisation and to represent realistic loading. In-service substation monitoring data was also obtained from Prof. Bortoni, in order to validate the methodology. Both methods are multivariate and utilise data from past time steps, they are trained on data and then tested on unseen data. The methods were adapted for application in continuous monitoring schemes in substations, either on fixed or robotic platforms. Both sets of results showed general improvements on methods previously in the literature. The quality of the results presented suggest that smart systems utilising thermal cameras, environmental data and load data, placed permanently in substations, would be capable of predicting future thermal output for a region of interest. Future work aims to utilise these predictions for fault detection.

\section{ACKNOWLEDGMENTS}

The authors would like to thank National Grid, for sponsoring and supporting the project and ESPRC, for funding the project.

\section{REFERENCES}

[1] Bortoni, E. d. C., Santos, L. d., Bastos, G. S., Souza, L. E. d., and Craveiro, M. A. C., "Extracting Load Current Influence From Infrared Thermal Inspections," IEEE Transactions on Power Delivery 26, 501-506 (Apr. 2011).

[2] Bortoni, E. C., Santos, L., and Bastos, G. S., "A Model to Extract Wind Influence From Outdoor IR Thermal Inspections," IEEE Transactions on Power Delivery 28, 1969-1970 (July 2013).

[3] Santos, L. d., Bortoni, E. C., Souza, L. E., Bastos, G. S., and Craveiro, M. a. C., "Infrared thermography applied for outdoor power substations," in [Thermosense XXX], 6939, 69390R, International Society for Optics and Photonics (Mar. 2008).

[4] Hochreiter, S. and Schmidhuber, J., "Long Short-Term Memory," Neural Comput. 9, 1735-1780 (Nov. 1997).

[5] Srivastava, N., Hinton, G., Krizhevsky, A., Sutskever, I., and Salakhutdinov, R., "Dropout: A Simple Way to Prevent Neural Networks from Overfitting," Journal of Machine Learning Research 15, 1929-1958 (2014). 